\title{
Dewetting Transitions of Au/Ni Bilayer Films
}

\author{
Xi Cen*, Andrew Thron, Xinming Zhang, Klaus van Benthem
}

Department of Chemical Engineering and Materials Science, One Shields Avenue, University of California, Davis, CA 95616

Thin films deposited at low temperatures are often kinetically constrained and will dewet the underlying substrate when heat-treated. Dewetting can be a serious concern in microelectronics reliability [1], while it can also be utilized for engineering of nanostructures with potentials in storage [2], catalysis [3], or optical/magnetic applications [4]. Mechanisms for dewetting of single layer films have been studied extensively. However little work has been reported on the cross-sectional characterization of dewetting processes for multilayer or alloyed thin films.

In this study, we report cross-sectional characterization of $\mathrm{Au} / \mathrm{Ni}$ bilayer dewetting at early stage. Changes in the morphology and chemistry were observed by cross-sectional transmission electron microscopy (TEM) techniques, including scanning transmission electron microscopy (STEM), Procession Electron Diffraction (PED), electron energy-loss spectroscopy (EELS) and energy-dispersive X-ray spectroscopy (EDXS).

$\mathrm{Ni}$ and $\mathrm{Au}$ films each with a nominal thickness of $30 \mathrm{~nm}$ were subsequently sputtered on $\mathrm{SiO}_{2} / \mathrm{Si}$ substrates. The bilayer films were then annealed at $675^{\circ} \mathrm{C}$ for 1 hour in vacuum at a base pressure of $6 \times 10^{-6}$ torr. Fast heating and cooling were achieved by pushing and withdrawing the quartz tube containing the samples into and out of the hot zone of the furnace. Cross-sectional TEM specimens were prepared by standard FIB lift-out techniques [5].

The early dewetting stage revealed obvious morphology and chemical distribution change, strong texture formation and void nucleation at preferential sites. Fig 1a shows a bright field TEM micrograph of as-deposited film. An abrupt change in contrast at the Ni/Au interface indicates that Ni and Au were well separated before annealing. After annealing (Fig 1b), Au and Ni grains rearranged and mixed as a semi-continuous film with thickness around $70 \mathrm{~nm}$. The majority of the metal $/ \mathrm{SiO}_{2}$ interface was comprised of $\mathrm{Au} / \mathrm{SiO}_{2}$ after annealing despite the initial deposition sequence of the as-deposited $\mathrm{Au} / \mathrm{Ni} / \mathrm{SiO}_{2} / \mathrm{Si}$ interface structure. Both $\mathrm{Au}$ and $\mathrm{Ni}$ show strong texture along the direction normal to the interface after annealing. Void formation is identified to be the mechanism in the Au/Ni bilayer dewetting process, rather than grain boundary grooving at the free surface of the metal film. STEM image (Fig 2a) and EDXS elemental mapping of Au (Fig 2b) and Ni (Fig 2c) showed the void formation at $\mathrm{Ni} / \mathrm{Au}$ grain boundaries and $\mathrm{Ni} / \mathrm{Au} / \mathrm{SiO}_{2}$ triple junctions, which were observed as preferential nucleation sites.

In-situ heating studies with TEM/STEM are currently underway to further investigate the dewetting kinetics of $\mathrm{Au} / \mathrm{Ni}$ bilayer films at the atomic length-scale. Preliminary results will be reported during the presentation. [6] 


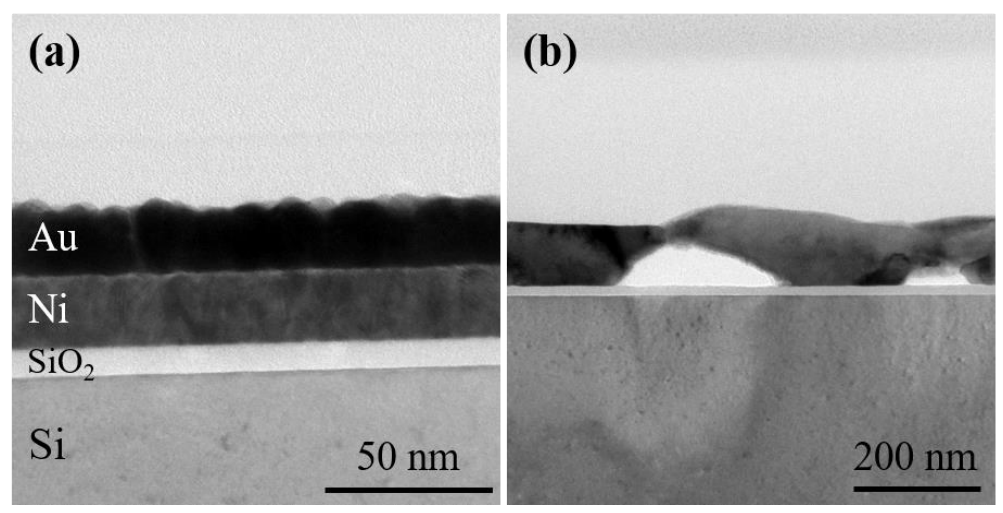

Figure 1. (a) Cross-sectional TEM bright field micrograph of the as-deposited bilayer film (b) Crosssectional TEM bright field micrograph of the annealed bilayer film.

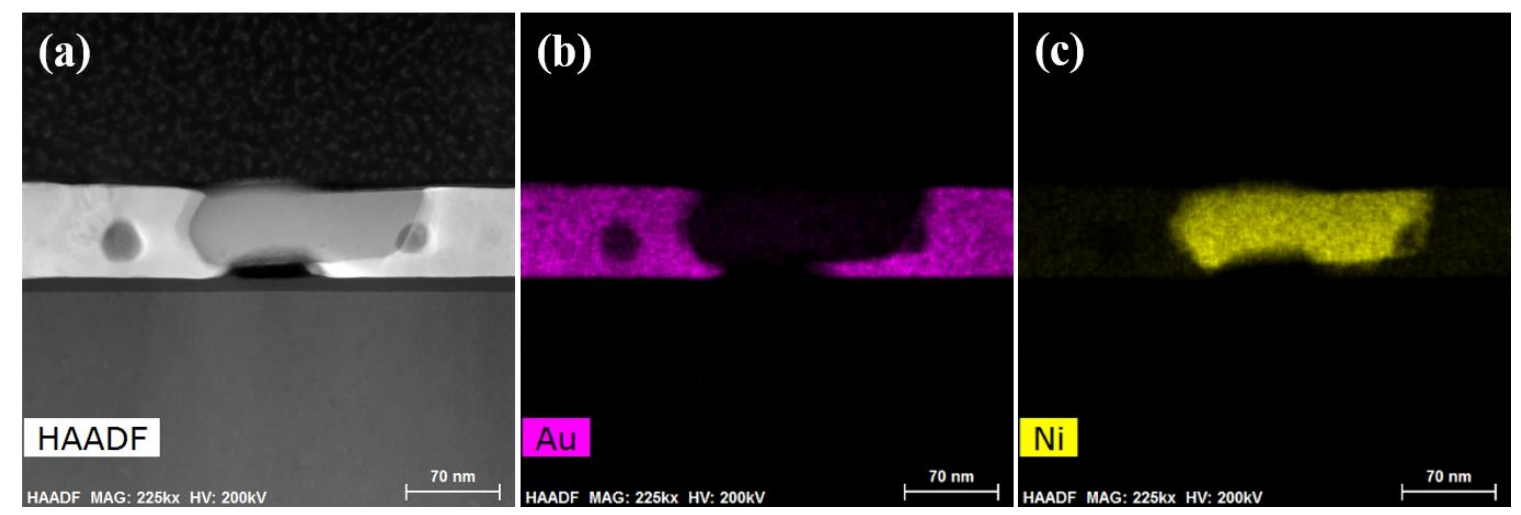

Figure 2. EDXS chemical distribution maps and corresponding STEM survey image. (a) is the STEM survey micrograph. (b) and (c) are EDXS chemical distribution maps obtained from spectral intensities of the $\mathrm{Au} \mathrm{M} \alpha_{1}$ (purple) and Ni L $\alpha_{1 \_2}$ (yellow).

\section{References:}

[1] D. Deduytsche et al, Journal of Applied Physics 98 (2005), 033526

[2] J.H. Chen et al, Electrochemical and Solid State Letters 10 (2007), H302-H304

[3] Y.F. Guan et al, Nanotechnology 19 (2008), 23560

[4] L. Armelao et al, Coordination Chemistry Reviews 250 (2006), 1294-1314

[5] A. Strecker et al, Zeitschrift für Metallkunde 94 (2003), 290-297

[6] This study was financially supported by a Faculty Early Career award from the US National Science Foundation (DMR-0955638). 